\title{
Patterns of obesity in boys and girls after treatment for acute lymphoblastic leukaemia
}

\author{
I Odame, J J Reilly, B E S Gibson, M D C Donaldson
}

\begin{abstract}
The frequency and pattern of obesity in survivors of acute lymphoblastic leukaemia (ALL) was examined in a retrospective analysis of height and weight at zero, two, and four years from diagnosis in 40 children (19 boys and 21 girls). The children had been treated according to the Medical Research Council protocols UKALL VIII and $X$, both of which included cranial radiotherapy at a dose of $1800 \mathrm{cGy}$. Body mass index (BMI), determined as weight/height ${ }^{2}$, was used as a measure of fatness. The BMI Z scores were calculated for each patient from standard tables. The ALL group was compared with a control group of 18 age matched children who had received chemotherapy but no radiotherapy. Changes in BMI between diagnosis and two and four years later were analysed by paired $t$ tests.
\end{abstract}

Mean BMI $Z$ scores at diagnosis were similar between ALL boys, ALL girls, and the control group. Two years after diagnosis the ALL group, particularly the girls, showed a significant increase in BMI. By four years BMI had decreased slightly in the ALL boys, but had increased still further in the ALL girls with $57 \%$ having $B M I Z$ scores greater than 2. In the control group BMI increased, but not significantly, at two and four years.

It is concluded that the obesity seen in patients treated for ALL is more pronounced in girls than boys, and that cranial irradiation is an important factor. (Arch Dis Child 1994; 71: 147-149)

Acute lymphoblastic leukaemia (ALL) is the most common childhood malignancy, affecting about one in 3500 children under 10 years in the United Kingdom. ${ }^{1}$ With current Medical Research Council (MRC) chemotherapy regimens, it is expected that $65-70 \%$ of affected children will achieve long term survival. ${ }^{2}$ Improved survival rates have focused attention on late effects including cardiotoxicity, ${ }^{3}$ educational and psychological dysfunction, ${ }^{4}$ secondary malignancy, ${ }^{5}$ and disorders of growth and puberty. ${ }^{6}$

The well documented effects of leukaemia treatment on linear growth and puberty relate largely to the dose of cranial irradiation received. Doses of 2400-2500 cGy cause growth hormone deficiency, ${ }^{7}$ often with reasonable growth during childhood, ${ }^{8}$ but attenuation of the pubertal growth spurt. ${ }^{9}$ Girls show a strong tendency towards precocious and early puberty. ${ }^{10}$ Cranial irradiation with 1800 cGy is associated with normal spontaneous growth hormone secretion in prepubertal subjects, but reduced secretion with an abnormal pattern at puberty. ${ }^{11}$

The prevalence of obesity in children treated for ALL is less well documented. Zee and Chen, in a large retrospective study of ALL survivors, confirmed the impression that ALL survivors tend to be overweight. ${ }^{12}$ Their group were heterogenous in terms of treatment regimen, however, had mostly received 2400 cGy for cranial treatment, and the obesity patterns in girls and boys were not examined separately. Moreover, normal data for body mass index (BMI) in children under the age of 6 years were unavailable at the time of their study. The aim of our study was to examine a group of ALL survivors in first remission, who had received cranial irradiation at $1800 \mathrm{cGy}$ according to UKALL VIII or X protocols, to look specifically for sex differences, and to compare the patterns of obesity with a control group who had received chemotherapy for a range of other malignant disorders, but no radiotherapy.

\section{Methods}

The medical records of children with ALL treated according to MRC UKALL VIII and X protocols presenting between the ages of 2 and 8 years and completing treatment between 1980 and 1990 were examined retrospectively. These protocols had a common remission induction treatment, cranial irradiation (1800 cGy in 10 fractions), with intrathecal methotrexate and maintenance chemotherapy for two years. In addition, the MRC UKALL $\mathrm{X}$ protocol included one or two blocks of intensive chemotherapy for some children. Of the 40 children in the study, 15 children (six boys and nine girls) were treated with UKALL VIII and 25 children (13 boys and 12 girls) according to UKALL $X$ protocol. Children who relapsed and received further chemotherapy, craniospinal, or testicular irradiation, or bone marrow transplantation were excluded. Thus all analysed children were in continued first remission.

To assess the role of cranial irradiation in the causation of obesity, we compared the ALL group with 18 age matched children (eight boys and 10 girls) who had received chemotherapy with or without an operation, but not radiotherapy. Their diagnoses were Wilms' tumour (six), non-Hodgkin's lymphoma (four), rhabdomyosarcoma (three), neuroblastoma (two), histiocytosis-X with systemic disease (two), and yolk sac tumour (one). 
Table 1 Changes in BMI Z score: to two and four years after diagnosis

\begin{tabular}{|c|c|c|c|c|}
\hline & \multicolumn{2}{|l|}{$A L L$ group } & \multicolumn{2}{|l|}{ Control group } \\
\hline & Girls & Boys & Girls & Boys \\
\hline \multicolumn{5}{|l|}{ Two years: } \\
\hline $\begin{array}{l}\text { Mean (SD) BMI Z score } \\
95 \% \text { Confidence interval }\end{array}$ & $1.87(1.03) \dagger$ & $1.39(1.40) \dagger$ & $0.93(1.79)(\mathrm{NS})$ & $-0.51(1.27)(\mathrm{NS})$ \\
\hline \multicolumn{5}{|l|}{ Four years: } \\
\hline $\begin{array}{l}\text { Mean (SD) BMI Z score } \\
95 \% \text { Confidence interval }\end{array}$ & $\begin{array}{l}2.34(1.36) \dagger \\
1.70 \text { to } 2.97\end{array}$ & $\begin{array}{l}1.23(1.49)^{\star} \\
0.51 \text { to } 1.94\end{array}$ & $\begin{array}{l}1.28(1.94) \text { (NS) } \\
-0.23 \text { to } 2.78\end{array}$ & $\begin{array}{l}0.17(1.12)(\mathrm{NS}) \\
-1.21 \text { to } 0.87\end{array}$ \\
\hline
\end{tabular}

$\mathrm{NS}=$ not statistically significant. ${ }^{\star}$ Statistically significant (paired $\left.t, \mathrm{p}<0 \cdot 05\right)$. †Statistically significant (paired $\left.t, \mathrm{p}<0 \cdot 001\right)$

Heights (measured with a Harpenden stadiometer) and weights were recorded at diagnosis and at all clinic visits thereafter. From 1989 onwards all children with ALL who had completed treatment and were older than 5 years were seen in a joint leukaemia/endocrine clinic, and pubertal data were available in these subjects. Onset of puberty was taken as the age of initial breast development in girls and testicular enlargement to $4 \mathrm{ml}$ in boys.

Body mass index was used as an index of fatness, according to the formula weight/height ${ }^{2}$. The BMI $\mathrm{Z}$ scores for each patient at zero (diagnosis), two, and four years were calculated with reference to the French population standards published by RollandCachera et al. ${ }^{13}$ The $\mathrm{Z}$ scores were calculated using a formula devised by these workers which takes into account changes in the skewness of the distribution of BMI data in relation to age. The changes in BMI $\mathrm{Z}$ scores from diagnosis to two and four years in each child were analysed by the paired $t$ test. Changes within each group of children were expressed as $95 \%$ confidence intervals.

\section{Results}

The mean (range) age at diagnosis was 3.4 $(2 \cdot 1-5 \cdot 2)$ and $3 \cdot 6(2 \cdot 0-6 \cdot 9)$ years respectively in 19 boys and 21 girls with ALL; $3.9(2 \cdot 8-7 \cdot 3)$ and $3 \cdot 7(2 \cdot 8-8 \cdot 1)$ years in eight control boys and 10 control girls. There was no significant difference between the groups in age and sex distribution.

Puberty was established but onset unknown in three girls with ALL and no data were available in one girl. Of the remaining 16 girls, two were still prepubertal and onset of puberty was $8 \cdot 0-10 \cdot 7$ years (median $10 \cdot 0$ ) in 14 . Puberty was established but onset unknown in two boys: no data were available in another two. Of the remaining 15 boys, 10 aged $6 \cdot 7-12 \cdot 5$ years were still prepubertal, while the onset of puberty was $9 \cdot 7-12.5$ years in the other five. Taking into account the ages at diagnosis of

Table 2 Absolute BMI $Z$ scores and prevalence of obesity at diagnosis and two and four years after diagnosis

\begin{tabular}{|c|c|c|c|c|}
\hline & \multicolumn{2}{|l|}{$A L L$ group } & \multicolumn{2}{|l|}{ Control group } \\
\hline & Girls & Boys & Girls & Boys \\
\hline $\begin{array}{l}\text { Diagnosis: } \\
\text { Mean (SD) BMI Z score } \\
\% \text { Obese }\end{array}$ & $\begin{array}{l}0.07(1 \cdot 20) \\
5\end{array}$ & $\begin{array}{l}-0.09 \\
5\end{array}(1.01)$ & $\begin{array}{l}-0.31(0.88) \\
10\end{array}$ & $\begin{array}{l}0.83(0.95) \\
0\end{array}$ \\
\hline $\begin{array}{l}\text { Two years: } \\
\text { Mean (SD) BMI Z score } \\
\text { \% Obese }\end{array}$ & $4^{1.93(1.09)}$ & ${ }_{26}^{1 \cdot 30(1 \cdot 11)}$ & $\begin{array}{l}0.62(1.44) \\
20\end{array}$ & $\begin{array}{l}0.12(1.03) \\
0\end{array}$ \\
\hline $\begin{array}{l}\text { Four years: } \\
\text { Mean (SD) BMI Z score } \\
\% \text { Obese }\end{array}$ & $\begin{array}{l}2 \cdot 36(1 \cdot 20) \\
57\end{array}$ & $21 \cdot 13(1 \cdot 25)$ & $20^{0.82(1.49)}$ & 25 \\
\hline
\end{tabular}

the three girls and four boys in whom the onset of puberty was unknown, we judged that two girls and one boy could have been in puberty two years after diagnosis, and that five girls and four boys were, or could have been, in puberty at four years. The difference in BMI Z scores for each group before and after eliminating those subjects who were, or could have been, pubertal was negligible.

Table 1 shows the highly significant change in BMI Z score in girls with ALL at two years, becoming even more marked at four years. In the boys with ALL, the change was significant but less dramatic at two years, and less marked, although still significant, at four years. By contrast, the control boys showed no change in mean BMI Z score over the four years. The control girls, on the other hand, showed a progressive increase at two and four years, but this was not significant. Table 2 shows the marked increase in prevalence of obesity in the girls with ALL, which reached $57 \%$ by four years, but which was similar (20-25\%) among the boys with ALL and the control group by this stage.

\section{Discussion}

This study confirms previous reports of obesity in ALL survivors, but indicates that the problem is mainly in girls. Early puberty could not be invoked as a significant factor as few patients were pubertal during the study period, and eliminating those who were or could have been pubertal had no impact on the BMI Z scores. Although the boys with ALL showed a significant increase in fatness at two years, the effect appeared to be waning by four years, in contrast with the girls with ALL. Although not significant, there was an increase in the fatness of control girls. Also, the prevalence of obesity in control boys was higher at four years than at diagnosis, although the mean value had changed little. These changes could be artefact, possibly reflecting differences between French and Scottish standards, or alternatively a late effect of the chemotherapy with the number of patients too small to show a significant trend.

Cranial irradiation is the most likely factor to account for the difference in obesity patterns between ALL and control subjects. Dysfunctional growth hormone release ${ }^{11}$ and early puberty ${ }^{10}$ are both manifestations of radiation induced hypothalamic dysfunction and it is probably relevant that girls are more vulnerable than boys.

In the current MRC UKALL XI trial, most children are receiving intrathecal methotrexate 
with or without high dose intravenous methotrexate rather than cranial irradiation. A prospective analysis of obesity patterns in the long term survivors from this trial should clarify the respective roles of cranial irradiation and chemotherapy in causing this problem. Meanwhile, studies on body composition and energy expenditure in obese ALL survivors are justified in an attempt to establish the mechanisms for, and possible management of, this late effect of cancer treatment.

1 Parkin DM, Stiller CA, Draper GJ, Bieber CA. The international incidence of childhood cancer. Int $\mathcal{f}$ Cancer 1988 42: 511-20.

2 Stiller CA, Bunch KJ. Trends in childhood cancer survival in Britain 1971-1985. Br f Cancer 1990; 62: 806-15.

3 Lipshultz SE, Colan SD, Gelber RD, Perez-Atayde AR, Sallan SE, Sanders SP. Late cardiac effects of doxorubicin therapy for acute lymphoblastic leukaemia in childhood. therapy for acute lymphoblastic leuk

4 Cousens P, Waters B, Said J, Stevens M. The cognitive effects of cranial irradiation in leukaemia: a survey and meta-analysis. f Clin Psychol Psychiatry 1988; 29: 839-52.
5 Neglia JP, Meadows AT, Robinson LL, et al. Second neoplasms after acute lymphoblastic leukaemia in childhood. N Engl f Med 1991; 325: 1330-6.

6 Swift PGF, Kearney PJ, Dalton RG, Bullimore JA, Mott MG, Savage DCL. Growth and hormonal status of children treated for acute lymphoblastic leukaemia. Arch Dis Child 1978; 53: 890-4.

7 Kirk JA, Ragnupathy P, Stevens MM, et al. Growth failure and growth-hormone deficiency after treatment for acute lymphoblastic leukaemia. Lancet 1987; i: 190-3.

8 Shalet SM, Price DA, Beardwell CG, Morris Jones PH Pearson D. Normal growth despite abnormalities of growth hormone secretion in children treated for acute growth hormone secretion in children

9 Moell C, Garwicz S, Westgren U, Wiebe T, AlbertssonWiklund K. Suppressed spontaneous secretion of growth hormone in girls after treatment for acute lymphoblastic leukaemia Arch Dis Child 1989; 64: 252-8.

10 Leiper AD, Stanhope R, Kitching P, Chessells JM. Precocious puberty and premature puberty associated with the treatment of acute lymphoblastic leukaemia. Arch Dis Child 1987; 62: 1107-12.

11 Crowne EC, Moore C, Wallace WHB, et al. A novel variant of growth hormone (GH) insufficiency following low dose cranial irradiation Clin Endocrinol (Oxf 1992; 36:59-68. therapy for acute lymphoblastic leukaemia. Am $\mathcal{f}$ Pediatr therapy for acute lymphoblas

13 Rolland-Cachera MF, Cole TJ, Sempe M, Tichet J, Rossignol C, Charraud A. Body mass index variations: centiles from birth to 87 years. Eur f Clin Nutr 1991; 45: 13-21 